\title{
Quadratic Upper Bounds on the Erdős-Pósa property for a generalization of Packing and Covering cycles
}

\author{
Fedor V. Fomin* ${ }^{*}$ Daniel Lokshtanov ${ }^{\dagger} \quad$ Neeldhara Misra $^{\ddagger}$ \\ Geevarghese Philip ${ }^{\ddagger} \quad$ Saket Saurabh ${ }^{\ddagger}$
}

May 19, 2014

\begin{abstract}
According to the classical Erdős-Pósa theorem, given a positive integer $k$, every graph $G$ either contains $k$ vertex disjoint cycles or a set of at most $\mathcal{O}(k \log k)$ vertices that hits all its cycles. Robertson and Seymour [Graph minors. V. Excluding a planar graph. J. Comb. Theory Series B, 41:92-114, 1986] generalized this result in the best possible way. More specifically, they showed that if $\mathcal{H}$ is the class of all graphs that can be contracted to a fixed planar graph $H$, then every graph $G$ either contains a set of $k$ vertex-disjoint subgraphs of $G$, such that each of these subgraphs is isomorphic to some graph in $\mathcal{H}$ or there exists a set $S$ of at most $f(k)$ vertices such that $G \backslash S$ contains no subgraph isomorphic to any graph in $\mathcal{H}$. However the function $f$ is exponential. In this note, we prove that this function becomes quadratic when $\mathcal{H}$ consists all graphs that can be contracted to a fixed planar graph $\theta_{c}$. For a fixed $c, \theta_{c}$ is the graph with two vertices and $c \geq 1$ parallel edges. Observe that for $c=2$ this corresponds to the classical Erdős-Pósa theorem.
\end{abstract}

\section{Introduction}

Given a graph $G$ we denote by $V(G)$ and $E(G)$ its vertex and edge set respectively. Let $G$ be a graph, and let $\mathcal{H}$ be a class of graphs. A $\mathcal{H}$-packing is a set of vertexdisjoint subgraphs of $G$ such that each of these subgraphs is isomorphic to some graph in $\mathcal{H}$. Similarly, a subset of vertices $S \subseteq V(G)$ is called an $\mathcal{H}$-cover if $G \backslash S$ contains no subgraph isomorphic to any graph in $\mathcal{H}$. The class $\mathcal{H}$ is said to have the Erdős-Pósa property for some graph class $\mathcal{G}$ if there exists a function $f: \mathbb{N} \rightarrow \mathbb{N}$ such that, for every $k \geq 0$, every graph $G \in \mathcal{G}$ either contains an $\mathcal{H}$-packing of size at least $k$, or has an $\mathcal{H}$-cover of size at most $f(k)$.

\footnotetext{
${ }^{*}$ Department of Informatics, University of Bergen, N-5020 Bergen, Norway. fomin@ii.uib.no.

${ }^{\dagger}$ University of California, San Diego, La Jolla, CA 92093-0404, USA, dlokshtanov@cs . ucsd.edu

${ }^{\ddagger}$ The Institute of Mathematical Sciences, Chennai - 600113, India. \{neeldhara|gphilip|saket\}@imsc.res.in.
} 
Erdős and Pósa [7] proved that the Erdős-Pósa property holds for all graphs when $\mathcal{H}$ is the class of all cycles. The problem of identifying more general graph classes where the Erdős-Pósa property is satisfied has attracted a lot of attention $[2,4,11,13,17,18]$. Extensions of this problem defined on matroids have also been investigated $[9,10]$.

Our discussions are concerned with graphs that are permitted to have parallel edges, that is, multiple edges with the same endpoints. While such structures are called multigraphs to distinguish them from simple graphs, we will continue to use the term "graph" with the implicit understanding that parallel edges are allowed.

The operation of contracting an edge $e=(u, v)$ in a graph $G$ results in a graph $G^{\prime}$, in which $u$ and $v$ are replaced by a new vertex $v_{e}$ and in which for every neighbour $w$ of $u$ or $v$ in $G$, there is an edge $\left(w, v_{e}\right)$ in $G^{\prime}$. If a contraction operation results in more than one edge between a pair of vertices, then we retain all the multiple edges in the resulting graph. We say that a graph $G$ can be contracted to a graph $H$ if $H$ can be obtained from $G$ by a series of edge contractions. We say that $H$ is a minor of $G$ if some subgraph $\tilde{G}$ of $G$ can be contracted to $H$; such a $\tilde{G}$ is called an $H$-minor model of $G$. A graph class $\mathcal{G}$ is minor-closed if any minor of a graph in $\mathcal{G}$ is again a member of $\mathcal{G}$.

For a fixed graph $H$, the class $\mathcal{H}=\mathcal{M H}$ consists of graphs that can be contracted to $H$. For a fixed $c$, let $\theta_{c}$ be the graph with two vertices and $c \geq 1$ parallel edges. Observe that for $H=\theta_{1}$, and $H=\theta_{2}, \mathcal{H}=\mathcal{M H}$ consists of all graphs that contain at least one edge and all graphs that contain at least one cycle, respectively. Robertson and Seymour [15, Proposition 8.2] proved the following seminal result.

Proposition 1. MH satisfies the Erdős-Pósa property for all graphs if and only if $H$ is planar.

See the monograph "Graph Theory" by R. Diestel [5, Corollary 12.4.10 and Exercise 39] for an alternate proof of Proposition 1, with the additional assumption that $H$ is connected. The bounding function $f(k)$ in the Erdős-Pósa property, as obtained in the different proofs of Proposition 1, is exponential in $k$. Fomin et al. [8] showed that the bound becomes linear for any connected planar graph $H$ when the graph class $\mathcal{G}$ is any non trivial minor-closed class, and a result of Birmelé et al demonstrates a quadratic bound for the class of all graphs when $H$ is a cycle of fixed length [2]. Also, the classical result of Erdős-Pósa [7] shows that the class $\mathcal{H}=\mathcal{M} \theta_{2}$ (the family of all cycles) has the Erdős-Pósa property with $f(k)=O(k \log k)$ when $\mathcal{G}$ is the set of all graphs. In this note we prove a quadratic bound for the case when $\mathcal{G}$ consists of all graphs and $\mathcal{H}$ consists of all graphs which can be contracted to a fixed planar graph $\theta_{c}$. Observe that for $c=2$ this corresponds to classical Erdős-Pósa theorem, albeit with a larger bound. Given a graph $G$ and a vertex subset $S \subseteq V(G)$, we call a set $S$ a $\theta_{c}$-hitting set if $G \backslash S$ does not contain $\theta_{c}$ as a minor. The main result of this paper is:

Theorem 1. [Erdős-Pósa property for $\theta_{c}$ ] For any fixed $c \in \mathbb{N}$, every graph $G$ either 
contains $k$ vertex-disjoint $\theta_{c}$-minor models, or has a $\theta_{c}$-hitting set of size at most $f(k)=O\left(k^{2}\right)$.

\section{The Erdős-Pósa Property for $\theta_{c}$}

In this section we give the proof of Theorem 1. Towards this we need following definitions.

Let $G$ be a graph. A tree decomposition of a graph $G$ is a pair $\left(T, \mathcal{X}=\left\{X_{t}\right\}_{t \in V(T)}\right)$ such that

- $\cup_{t \in V(T)} X_{t}=V(G)$,

- for every edge $(x, y) \in E(G)$ there is a $t \in V(T)$ such that $x, y \subseteq X_{t}$, and

- for every vertex $v \in V(G)$ the subgraph of $T$ induced by the set $\left\{t \mid v \in X_{t}\right\}$ is connected.

The width of a tree decomposition is $\left(\max _{t \in V(T)}\left|X_{t}\right|\right)-1$ and the treewidth of $G$ is the minimum width over all tree decompositions of $G$. A tree decomposition $(T, \mathcal{X})$ is called a nice tree decomposition if $T$ is a tree rooted at some node $r$ where $X_{r}=\emptyset$, each node of $T$ has at most two children, and each node is of one of the following kinds:

1. Introduce node: a node $t$ that has only one child $t^{\prime}$ where $X_{t} \supset X_{t^{\prime}}$ and $\left|X_{t}\right|=\left|X_{t^{\prime}}\right|+1$.

2. Forget node: a node $t$ that has only one child $t^{\prime}$ where $X_{t} \subset X_{t^{\prime}}$ and $\left|X_{t}\right|=$ $\left|X_{t^{\prime}}\right|-1$.

3. Join node: a node $t$ with two children $t_{1}$ and $t_{2}$ such that $X_{t}=X_{t_{1}}=X_{t_{2}}$.

4. Base node: a node $t$ that is a leaf of $t$, is different than the root, and $X_{t}=\emptyset$.

Notice that, according to the above definition, the root $r$ of $T$ is either a forget node or a join node. It is well known that any tree decomposition of $G$ can be transformed into a nice tree decomposition maintaining the same width [12]. We use $G_{t}$ to denote the graph induced on the vertices $\cup_{t^{\prime}} X_{t}^{\prime}$, where $t^{\prime}$ ranges over all descendants of $t$, including $t$. We use $H_{t}$ to denote $G_{t}\left[V\left(G_{t}\right) \backslash X_{t}\right]$.

We prove Theorem 1 by establishing the following two lemmas.

Lemma 1. If the treewidth of a graph $G$ is at least $2 c^{2} k^{2}$, then $G$ contains at least $k$ vertex-disjoint $\theta_{c}$-minor models.

Lemma 2. If the treewidth of $G$ is at most $2 c^{2} k^{2}$ and $G$ does not contain $k$ vertexdisjoint $\theta_{c}$-minor models, then $G$ contains a $\theta_{c}$-hitting set of size at most $\eta k^{2}=$ $O\left(k^{2}\right)$, where the constant $\eta$ depends only on $c$.

The proof of Theorem 1 follows from the above two lemmas. 
Proof of Theorem 1. Suppose graph $G$ does not contain $k$ vertex-disjoint $\theta_{c}$-minor models. Then by Lemma $1, G$ has treewidth at most $2 c^{2} k^{2}$. Now by applying Lemma 2, we have that $G$ contains a $\theta_{c}$-hitting set of size $O\left(k^{2}\right)$.

We now define some terms which we use in the proof of Lemma 1. A bramble is a set of connected subgraphs, called the elements of the bramble, any two of which either intersect or are linked by at least one edge. A hitting set of a bramble is a set of vertices which meets every element of the bramble. The order of a bramble is the minimum cardinality of a hitting set of the bramble. The maximum order of a bramble in a graph is its bramble number. Brambles and tree decompositions are dual structures in the following sense.

Proposition 2. [16] The tree-width of any graph is exactly one less than its bramble number.

Our proof of Lemma 1 uses some ideas from the proof of Lemma 3.2 in Reed and Wood's recent work [14] on grid-like minors.

Lemma 3. [3] Let $\mathcal{B}$ be a bramble in a graph $G$. Then $G$ contains a path that intersects every element of $\mathcal{B}$.

Now we are ready to give a proof of Lemma 1.

Proof of Lemma 1. We show that if the treewidth of a graph $G$ is at least $2 c^{2} k^{2}$, then $G$ contains at least $k$ vertex-disjoint $\theta_{c}$-minor models. If the treewidth of $G$ is at least $2 c^{2} k^{2}$, then by Proposition 2, $G$ contains a bramble (call it $\mathcal{B}$ ) of order at least $2 c^{2} k^{2}+1$. By Lemma 3 , there exists a path that visits every element of the bramble at least once. Let $P$ be such a path, and let $v_{1}, \ldots, v_{t}$ be the vertices of $P$ (stated in the order of their appearance in $P$ ). Note that $t \geq 2 c^{2} k^{2}+1$, as otherwise $P$ would be a hitting set of $\mathcal{B}$ with fewer vertices than the order of $\mathcal{B}$.

For $1 \leq i \leq t$, let $B_{i}$ denote the set of all elements of $\mathcal{B}$ which contain the vertex $v_{i}$. Note that for $1 \leq i \leq t, \cup_{j=1}^{i} B_{j}$ is a bramble. That is, the family

$$
\mathcal{T}_{i}=\bigcup_{j=1}^{i} B_{j}=\left\{B \mid B \in \mathcal{B} \text { and } B \cap\left\{v_{1}, \ldots, v_{i}\right\} \neq \emptyset\right\}
$$

is a bramble. Let $O_{i}$ denote the order of this bramble. Let $s$ be the smallest number such that $O_{s}=c^{2} k^{2}$. The existence of such $s$ is guaranteed by the fact that $O_{1}=1$, $O_{t}>2 c^{2} k^{2}$, and for $1 \leq i \leq t-1, O_{i+1} \leq O_{i}+1$. Let $\mathcal{B}_{1}=\mathcal{T}_{s}$, and let $\mathcal{B}_{2}=\mathcal{B} \backslash \mathcal{B}_{1}$. Since the value of $O_{i}$ increases by at most one in a single step, we have that the order of $\mathcal{B}_{2}$ is at least $c^{2} k^{2}$ - indeed, if not, combining a smallest hitting set for $\mathcal{B}_{1}$ with one for $\mathcal{B}_{2}$ would give us a hitting set of $\mathcal{B}$ which is smaller than the order of $\mathcal{B}$, a contradiction. Let $P_{1}$ be the sub-path of $P$ starting at $v_{1}$ and ending at $v_{s}$, and $P_{2}$ the subpath starting at $v_{s+1}$ and ending at $v_{t}$. By the above argument, $P_{1}$ and $P_{2}$ contain at least $c^{2} k^{2}$ vertices each.

Now, there must exist a collection, say $\mathcal{P}$, of at least $c^{2} k^{2}$ vertex-disjoint paths that begin in $P_{1}$ and end in $P_{2}$. If not, then by Menger's theorem, there exists a 
$P_{1}-P_{2}$ separator, say $S$, of size less than $c^{2} k^{2}$. Note that $S$ cannot be a hitting set of the brambles $\mathcal{B}_{1}$ or $\mathcal{B}_{2}$, since the order of each of these is at least $c^{2} k^{2}$. So there exist elements $A \in \mathcal{B}_{1}, B \in \mathcal{B}_{2}$ such that $A \cap S=\emptyset=B \cap S$. But since $A$ and $B$ are connected subgraphs which either intersect or are linked by an edge - being elements of $\mathcal{B}$ - and $A \cap P_{1} \neq \emptyset, B \cap P_{2} \neq \emptyset, S$ cannot be a $P_{1}-P_{2}$ separator.

We now show that $\mathcal{P} \cup P_{1} \cup P_{2}$ contains $k$ vertex-disjoint $\theta_{c}$-minor models. Let $V_{p}$ be the set of vertices that form the end points (on $P_{1}$ and $P_{2}$ ) of the paths in $\mathcal{P}$. For $i \in\{1,2\}$, let $Q_{i}=P_{i} \cap V_{p}$. We label both $Q_{1}$ and $Q_{2}$ with a common index set $[M]$, where $M=\left|Q_{1}\right|=\left|Q_{2}\right|$. Let $f:[M] \rightarrow[M]$ be the following bijection: $f(i)=j$ if and only if there is a path in $\mathcal{P}$ that begins in $i$ and ends in $j$. We say that a subset of paths $C \subseteq \mathcal{P}$ is cross-free under this labeling if there does not exist $i, i^{\prime} \in Q_{1} \cap C ; i<i^{\prime}$ and $f(i)>f\left(i^{\prime}\right)$.

Note that since the paths in $\mathcal{P}$ are vertex-disjoint, the numbers $f(1), f(2), \ldots$, $f(M)$ form a permutation of $M$, and by the Erdős-Szekeres Theorem [6], the sequence $\langle f(1), f(2), \ldots, f(M)\rangle$ contains a monotonically increasing or decreasing subsequence of length at least $t$, where $t$ is $\sqrt{|M|}=c k$. Let a witness subsequence be $\left\langle f\left(s_{1}\right), f\left(s_{2}\right), \ldots, f\left(s_{t}\right)\right\rangle$. Let $Q_{1}^{\prime}=\left\{s_{1}, s_{2}, \ldots, s_{t}\right\}$ and $Q_{2}^{\prime}=\left\{f\left(s_{1}\right), f\left(s_{2}\right), \ldots\right.$, $\left.f\left(s_{t}\right)\right\}$. Then the paths in $\mathcal{P}$ that have their end points in $Q_{1}^{\prime}, Q_{2}^{\prime}$ form a crossfree collection. These paths together with $P_{1}, P_{2}$ contain at least $k$ vertex-disjoint $\theta_{c}$-minor models.

Before proving Lemma 2, we define the notion of a good labeling function. Given a nice tree decomposition $\left(T, \mathcal{X}=\left\{X_{t}\right\}_{t \in V(T)}\right)$ of a graph $G$, a function $g: V(T) \rightarrow \mathbb{N}$ is called a good labeling function if it satisfies the following properties:

- if $t$ is a base node then $g(t)=0$;

- if $t$ is an introduce node, then $g(t)=g(s)$, where $s$ is the child of $t$;

- if $t$ is a join node, then $g(t)=g\left(s_{1}\right)+g\left(s_{2}\right)$, where $s_{1}$ and $s_{2}$ are the children of $t$; and

- if $t$ is a forget node, then $g(t) \in\{g(s), g(s)+1\}$, where $s$ is the child of $t$.

Now we are ready to prove the covering lemma-Lemma 2.

Proof of Lemma 2. Here, we show that if $G$ has treewidth at most $2 c^{2} k^{2}$ and does not have more than $k-1$ disjoint minor models of $\theta_{c}$, then there exists a set $S \subseteq V(G),|S|=O\left(k^{2}\right)$, such that $G \backslash S$ does not contain $\theta_{c}$ as a minor.

Consider a nice tree decomposition $\left(T, \mathcal{X}=\left\{X_{t}\right\}_{t \in V(T)}\right)$ of the graph of width at most $2 c^{2} k^{2}$. Recall that for $t \in V(T), G_{t}$ is the graph induced on the vertices $\cup_{t^{\prime}} X_{t^{\prime}}$, where $t^{\prime}$ ranges over all descendants of $t$ including $t$, and $H_{t}$ is $G_{t} \backslash X_{t}$.

Let $P_{\theta_{c}}(G)$ denote the maximum number of vertex-disjoint $\theta_{c}$-minor models in $G$. We use $k^{\prime}$ to denote $P_{\theta_{c}}(G)$, and note that $k^{\prime} \leq k-1$.

Consider the function $\mu: V(T) \rightarrow\left[k^{\prime}\right]$, defined as follows: $\mu(t)=P_{\theta_{c}}\left(H_{t}\right)$. The function $\mu$ is a good labeling function because:

- If $t$ is a base node then $\mu(t)=0$ as $H_{t}$ is an empty graph. 


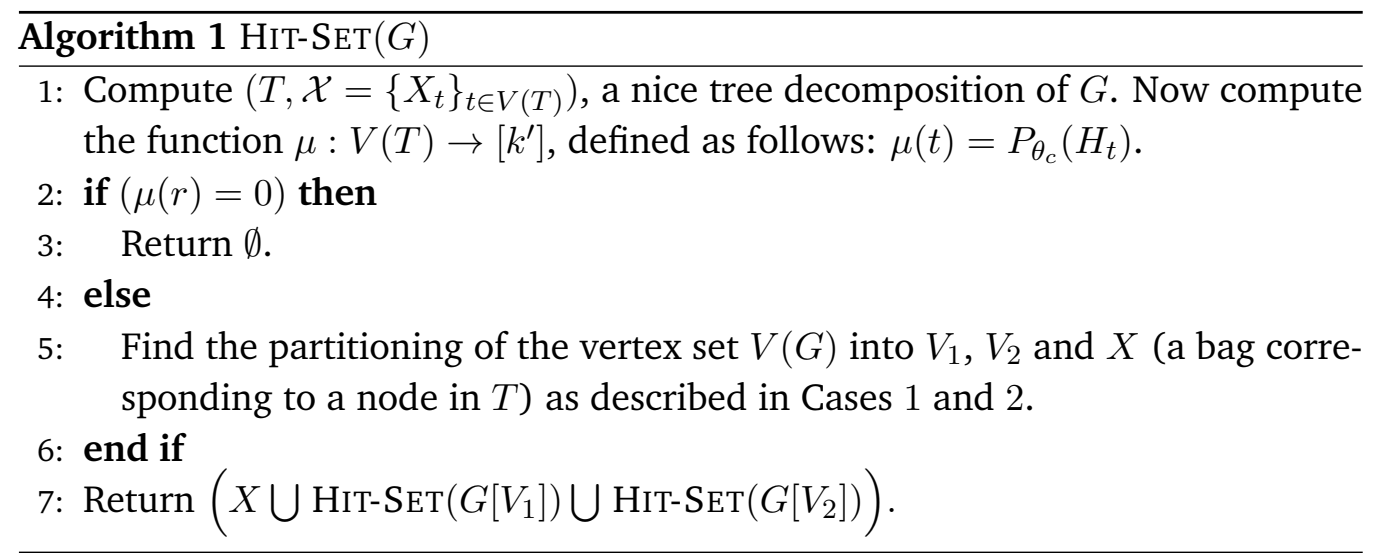

- If $t$ is an introduce node, then $\mu(t)=\mu(s)$, where $s$ is the child of $t$. Indeed, this follows from the fact that the graphs $H_{t}$ and $H_{s}$ are exactly the same.

- If $t$ is a join node, then $\mu(t)=\mu\left(s_{1}\right)+\mu\left(s_{2}\right)$, where $s_{1}$ and $s_{2}$ are the children of $t$. This follows from the fact that the bag $X_{t}$ is a separator of $G_{t}$ and $V\left(H_{s_{1}}\right) \cap V\left(H_{s_{2}}\right)=\emptyset$.

- If $t$ is a forget node, then $\mu(t) \in\{\mu(s), \mu(s)+1\}$, where $s$ is the child of $t$. This is because $H_{t}$ has at most one vertex more than $H_{s}$, which can add at most one to the number of vertex-disjoint $\theta_{c}$-minor models.

By definition, and by the convention that the bag $X_{r}$ corresponding to the root $r$ is $\emptyset$ we have that $\mu(r)=k^{\prime}$. To find the desired $\theta_{c}$-hitting set we give a recursive algorithm. We find a bag $X$ in the given tree decomposition such that its removal allows us to decompose the graph into two parts such that there are no edges from one part to another and the number of vertex-disjoint minor models of $\theta_{c}$ in each part is essentially a constant fraction of the original. After this we find a $\theta_{c}$-hitting set in each of these graphs and then take the union of these sets, together with the bag we removed to get these graphs, to get the desired hitting set for the whole graph. Let $t \in V(T)$ be the node where $\mu(t)>2 k^{\prime} / 3$ and for each child $t^{\prime}$ of $t$, $\mu\left(t^{\prime}\right) \leq 2 k^{\prime} / 3$. From the definition of good labeling function, this node exists and is unique provided that $k^{\prime}>0$. Moreover, observe that $t$ could either be a forget node or a join node. We distinguish these two cases.

- Case 1. If $t$ is a forget node, we set $V_{1}=V\left(H_{t^{\prime}}\right)$ and $V_{2}=V(G) \backslash\left(V_{1} \cup X_{t^{\prime}}\right)$ and observe that $P_{\theta_{c}}\left(G\left[V_{i}\right]\right) \leq\left\lfloor 2 k^{\prime} / 3\right\rfloor, i=1,2$. Also we set $X=X_{t^{\prime}}$.

- Case 2. If $t$ is a join node with children $t_{1}$ and $t_{2}$, we have that $\mu\left(t_{i}\right) \leq$ $2 k^{\prime} / 3, i=1,2$. However, as $\mu\left(t_{1}\right)+\mu\left(t_{2}\right)>2 k^{\prime} / 3$, we also have that either $\mu\left(t_{1}\right) \geq k^{\prime} / 3$ or $\mu\left(t_{2}\right) \geq k^{\prime} / 3$. Without loss of generality we assume that $\mu\left(t_{1}\right) \geq k^{\prime} / 3$ and we set $V_{1}=V\left(H_{t_{1}}\right), V_{2}=V(G) \backslash\left(V_{1} \cup X_{t_{1}}\right)$ and $X=X_{t_{1}}$.

We present a detailed algorithm to find a $\theta_{c}$-hitting set in Algorithm 1. The algorithm $\operatorname{Hit-Set}(G)$ takes as input a graph $G$ and returns a $\theta_{c}$-hitting set for $G$. Now we bound the size of the $\theta_{c}$-hitting set returned by the algorithm. Let 
$\mathcal{S}\left(G, P_{\theta_{c}}(G)\right)=\mathcal{S}\left(G, k^{\prime}\right)$ be the size of the $\theta_{c}$-hitting set returned by Hit-SeT $(G)$, where the second parameter denotes the number of minor models of $\theta_{c}$ in $G$. Then the value of $\mathcal{S}\left(G, P_{\theta_{c}}(G)\right)$ is upper bounded by the following recurrence:

$$
\mathcal{S}\left(G, k^{\prime}\right) \leq \max _{1 / 3 \leq \alpha \leq 2 / 3}\left\{\mathcal{S}\left(G\left[V_{1}\right], \alpha k^{\prime}\right)+\mathcal{S}\left(G\left[V_{2}\right],(1-\alpha) k^{\prime}\right)+2 c^{2} k^{2}\right\}
$$

Note that $P_{\theta_{c}}\left(G\left[V_{1}\right]\right)+P_{\theta_{c}}\left(G\left[V_{2}\right]\right) \leq 2 P_{\theta_{c}}(G) / 3$. Recalling that $k^{\prime} \leq k$, it is easy to see that the above recurrence solves to $O\left(k^{2}\right)$ using the Akra-Bazzi Theorem [1]. This concludes the proof.

\section{Conclusion}

In this short note we obtained a quadratic upper bound on the Erdős-Pósa property of a generalization of packing and covering cycles. It follows from tight Erdős-Pósa bound on cycles that the quadratic upper bound obtained in this paper on a generalization of packing and covering cycles can not be improved beyond $O(k \log k)$. We believe that even for $\theta_{c}$, the correct upper bound on the size of a minimum hitting set when a graph $G$ does not have $k$ vertex disjoint $\theta_{c}$-minor models is $O(k \log k)$. An interesting question will be to classify those planar graphs $H$, such that $\mathcal{M H}$ has Erdős-Pósa property with a polynomial function on all graphs.

\section{References}

[1] Mohamad Akra and Louay Bazzi. On the solution of linear recurrence equations. Computational Optimization and Applications, 10(2):195-210, 1998.

[2] Etienne Birmelé, J. Adrian Bondy, and Bruce A. Reed. The Erdős-Pósa property for long circuits. Combinatorica, 27(2):135-145, 2007.

[3] Etienne Birmelé, John Adrian Bondy, and Bruce A. Reed. Brambles, prisms, and grids. In Graph theory in Paris, Trends Math., pages 37-44. Birkhäuser Verlag, 2007.

[4] I. J. Dejter and V. Neumann Lara. Unboundedness for generalized odd cyclic transversality. In Combinatorics (Eger, 1987), volume 52 of Colloq. Math. Soc. János Bolyai, pages 195-203. North-Holland, Amsterdam, 1988.

[5] Reinhard Diestel. Graph theory, volume 173 of Graduate Texts in Mathematics. Springer-Verlag, Berlin, third edition, 2005.

[6] P. Erdös and G. Szekeres. A combinatorial problem in geometry. Compositio Math., 2:463-470, 1935.

[7] Paul Erdős and Louis Pósa. On independent circuits contained in a graph. Canad. J. Math., 17:347-352, 1965. 
[8] Fedor V. Fomin, Saket Saurabh, and Dimitrios M. Thilikos. Strengthening Erdős-Pósa property for minor-closed graph classes. Journal of Graph Theory, 66(3):235-240, 2011.

[9] James F. Geelen, A. M. H. Gerards, and Geoff Whittle. Disjoint cocircuits in matroids with large rank. J. Combin. Theory Ser. B, 87(2):270-279, 2003.

[10] Jim Geelen and Kasper Kabell. The Erdős-Pósa property for matroid circuits. J. Combin. Theory Ser. B, 99(2):407-419, 2009.

[11] Ken-Ichi Kawarabayashi and Atsuhiro Nakamoto. The Erdős-Pósa property for vertex- and edge-disjoint odd cycles in graphs on orientable surfaces. Discrete Math., 307(6):764-768, 2007.

[12] T. Kloks. Treewidth. Computations and Approximations., volume 842. Springer, Lecture Notes in Computer Science, 1994.

[13] Dieter Rautenbach and Bruce Reed. The Erdős-Pósa property for odd cycles in highly connected graphs. Combinatorica, 21(2):267-278, 2001. Paul Erdős and his mathematics (Budapest, 1999).

[14] Bruce A. Reed and David R. Wood. Polynomial treewidth forces a large gridlike-minor, 2011. Published online. DOI : 10.1016/j.ejc.2011.09.004.

[15] Neil Robertson and P. D. Seymour. Graph minors. V. Excluding a planar graph. J. Comb. Theory Series B, 41:92-114, 1986.

[16] P. D. Seymour and Robin Thomas. Graph searching and a min-max theorem for tree-width. J. Combin. Theory Ser. B, 58(1):22-33, 1993.

[17] Carsten Thomassen. On the presence of disjoint subgraphs of a specified type. J. Graph Theory, 12(1):101-111, 1988.

[18] Carsten Thomassen. The Erdős-Pósa property for odd cycles in graphs of large connectivity. Combinatorica, 21(2):321-333, 2001. Paul Erdős and his mathematics (Budapest, 1999). 